\title{
Vehicle Transmission
}

National Cancer Institute

\section{Source}

National Cancer Institute. Vehicle Transmission. NCI Thesaurus. Code C128364.

The indirect transmission of an infectious agent to a host via inanimate objects such as food, water, biologic products, or fomites. 